\title{
GIS Application for Economic Assessment of Direct Disaster Losses
}

\author{
Dimitar Dimitrov, Georgi Penchev and Ekaterina Bogomilova \\ University of National and World Economy, 1700 Sofia, Bulgaria \\ dimdimeunwe.bg
}

\begin{abstract}
The article is aimed at presenting the proof-of-concept system for automated direct disaster losses, developed within a scientific project of University of National and World Economy, Sofia. The idea behind the project was to create a system that can be used by customers without expert knowledge on physical or economic modeling of disaster effects. Thus alleviating the initial phases of disaster planning in administration, providing raw picture of disaster threats. The article describes in general conceptual and physical schemes of the system and gives main requirements for data and GIS applications that can be used. The process of developing such system shows that it is a complicated, but possible task. The system is not production ready and it is implemented with one physical model for floods. Nevertheless, the possibility to use and reuse the physical model by automation of main model estimation phases gives opportunity for creation and assessment of different alternatives for disaster prevention and relief.
\end{abstract}

Keywords: GIS, Disaster Losses, Disaster Modeling.

\section{Introduction}

Under the conditions of increasing climate change, good resource management for the protection of the population in disasters and accidents is becoming an even more upto-date topic. Decisions on the allocation of defense costs should be made after a thorough analysis and public consultation.

At the same time, modeling and analysis of natural disasters, as well as construction of appropriate defense facilities, are activities that require a considerable amount of knowledge and experience. Such a level of expertise is difficult to ensure at all times, especially at the regional and municipal level of the state administration.

In this connection, the importance of creating and using GIS applications with a high degree of automation of the analysis process can be seen in all its stages - from physical modeling to economic analysis of alternatives for prevention and management of the consequences of natural disasters.

The purpose of this article is to present the possibilities of using GIS to standardize and automate the economic analysis of disasters and accidents.

The paper explores the results of a project work carried out at the University of National and World Economy to develop an integrated information system for economic 
analysis of the damage caused by natural disasters. The presented system is not a finished product ready for use but rather an example of proving the concept that it is possible to integrate and automate the analysis of prevention and the consequences of risk events using modern GIS technologies.

\section{Requirements to the System and Software Applications}

Economic effects of disasters can generally be described as direct and indirect effects.

Direct damages may be inflicted on immovable assets and on stock (including final goods, goods in process, raw materials, materials and spare parts). In essence, this category consists of damage to assets that occurred right at the time of the actual disaster. It may include the total or partial destruction of physical infrastructure, buildings, installations, machinery, equipment, means of transportation and storage, furniture, damage to farmland, irrigation works, reservoirs and the like. In the special case of agriculture, the destruction of crops ready for harvest must also be valued and included as direct damage.

Direct disaster losses refer to directly quantifiable losses such as the number of people killed and the damage to buildings, infrastructure and natural resources.

Indirect disaster losses include declines in output or revenue, and impact on wellbeing of people, and generally arise from disruptions to the flow of goods and services as a result of a disaster. The definitions of these concepts may fundamentally differ from one author to the other.

The proposed experimental integrated system focuses only on the determination of direct losses from potential disasters and accidents. The identification of indirect losses requires further analysis to determine the logistical connectivity between enterprises, the state of the transport network, the state and factors affecting the demand and supply of different products, the availability of substitute products, etc. Inclusion of a requirement for indirect cost analysis would lead to significant system complexity and the implementation of multiple additional analytical actions.

In addition, the direct effects and economic losses are estimated only on the basis of possible infrastructure damages.

The objective of accelerating and mitigating the work of the consumer analyzing the effects of natural disasters can be achieved if the following requirements are met:

- usability - the ability of the user to easily identify the region of research, the main characteristics of the terrain and the economic sites;

- standardization - using standards for information and protocols for the services provided. As a basis for these standards, those provided by Open Geospatial Consortium (OGC)[1] were chosen;

- automation - automating as much of the economic assessment as possible, starting with the choice of the region for evaluation, going through physical analysis and predicting the impact of the natural disaster, to the application of the cost model for economic evaluation of the affected sites; 
- the ability to use software and data with free licenses and free access. This requirement was established to protect against dependence on the software and the dependence of company decisions, which is one of the EU requirements in such cases.

The idea behind the creation of the system is the use of a web interface. This provides a number of advantages, such as simplifying the use of the system and significantly better control and security. Moreover, the use of web-based systems is in line with the established practice of leading countries, organizations and companies in this field.

\subsection{Conceptual Model}

Taking into account the fact that the disaster analysis system must have a high degree of autonomy and independence, it can not rely on information from general or global mapping systems. It is therefore necessary to set up a proprietary mapping and information system.

Another significant argument to the system was taken into account here - the system shall not only provide, but also analyze the user's requests with the highest possible degree of automation. This in turn means that the basics of the model must be placed in the "Methods and Analytical Applications" field in Fig. 1. In other words, the conceptual scheme of the system shall be tailored to the selected analytical software and from there compatible applications for visualization and presentation of the results shall be sought.

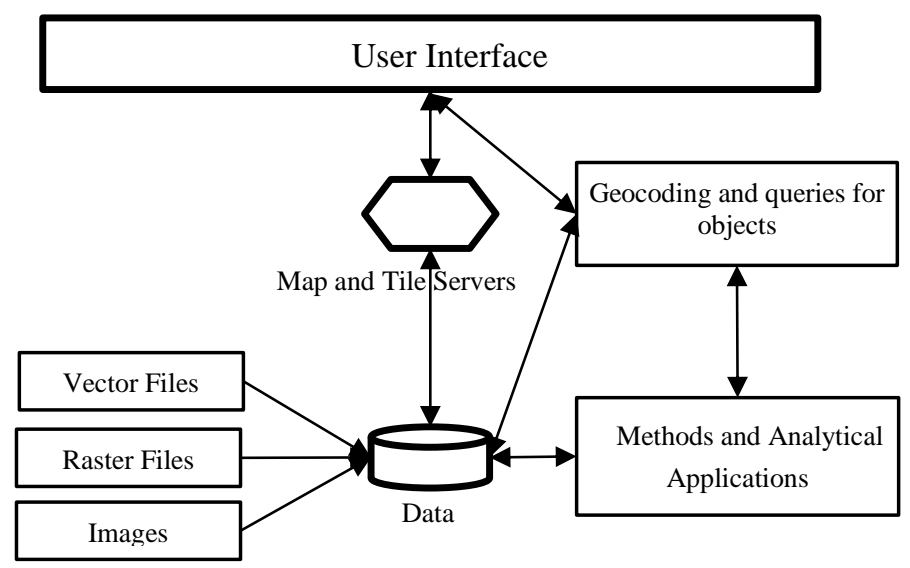

Fig. 1. Conceptual model of the integrated software system

Taking into account the system requirements described above, the GRASS GIS[2] application was selected. This is one of the open source software applications developed for the longest time, by a vast community of designers, and is based on perhaps the most powerful geographic software of general application - GDAL[3]. The GRASS 
GIS application was originally developed by the U.S. Army Construction Engineering Research Laboratories. There is hardly anything from the proprietary geographic software that these two applications cannot accomplish. Most of the methods described in the first stage of the study are implemented in GRAS GIS, and the rest can be implemented by GDAL, or another application using this library.

For the analysis, we need to have vector, raster and photo (orthographic or georeferenced satellite or aerial images) data. The latter are not so important for the analysis itself, but are important to the user who, by having them, can better navigate the research area.

The data should cover the entire region of a given country and, if possible, the neighboring countries (natural disasters are not limited by administrative boundaries). This is a huge amount of information and its presentation is impossible unless multiple-level maps are used. In popular geographic systems, this is called the zoom function, which allows the illusion of zooming in and out of objects, through detailing and aggregation. This feature is realized using the so-called Map Tile Servers. The use of tiles reflects exactly what these apps do - split or summarize the map into smaller or larger, equal in size to the visible tile region.

Mapnik[4], one of the most popular, fast and seriously developed open-source map applications, was selected for the developed system. Mod Tile[5] was chosen as a Tile Server for maps provided by Mapnik.

\subsection{Geocoding and References}

The installation and use of a map server do not exhaust the necessary services required for system operation. The map server actually provides maps based on raster or vector data in a photo format (usually png, jpeg, svg, or tiff) that are further stylized according to a predefined color scheme for the terrain, forests, roads, and so on. There is no way to perform search or interactive mapping in these formats. These two features require two additional software applications.

For the needs of the model, the following specialized applications were selected:

- R [6], Shiny [7] and Shiny Server [8] - the software platform and the statistical language $\mathrm{R}$ were chosen as a common software application management environment. Shiny Server was selected as a server application to be used in Shiny Web environment and its associated $\mathrm{R}$ packets, which should provide visualization of the results;

- Nominatim [9] - server for geocoding and search of objects by name;

- OverPass API [10] - Application programming interface (API) to search by object types.

The importance of such search for automating the analysis is obvious - the system must be able to quickly and accurately identify all important objects and classify them - for example, residential buildings, industrial buildings, shops, etc. 


\subsection{Data Sources}

Due to the fact that in Bulgaria there are practically no free available sources of official geographic information, the following global sources were used.

\section{Vector Files}

The only publicly available global vector data is provided by OpenStreetMap. This source uses "osm" format files based on the XML standard. Initially, information was introduced by volunteers - businesses and individuals. Later, municipalities and whole countries joined the system. Currently, most of the EU leading countries are to provide all non-classified geographic data in "osm" format.

\section{Raster Files}

The GISCO portal of the European Commission (the Geographical Information System of the COmmission)[11] is installed on the EUROSTAT website and provides publicly available information for the EU. For the purposes of this study, the files used are in raster format - improved and extrapolated Digital Elevation Model from Landsat capture with a resolution of about $27 \times 27$ meters of the tiff format cell for the EU member states.

Other relevant data sources for physical disaster modeling can also be the INSPIRE portal of the EC containing different environmental and geographic data of the EU Member States as follows:

- US Geographic Survey (USGS)[12] geographic portals provide vast geo-spatial information across the Earth in the form of a variety of raster formats;

- Joint Research Centre European Soil Data Centre (ESDAC)[13] provides data in a raster format about the soils on the entire land surface;

- the geo-referenced European Environment Agency's temperature database provides significant information in different geographic file formats about temperatures, winds, currents and pollution within the EU.

Information from the EU Copernicus Crisis Monitoring and Management Portal[14] can be used as a source for verification of the applied models.

\section{Working Process of the System}

The logical sequence of the workflow of the system can be summarized in three main stages - Select a region and create a physical disaster model, Determine losses, Analyze alternatives for prevention.

The chosen physical model for analysis is a 1D flood pattern. There are two main reasons for choosing this model.

First, the model only requires vector and raster data that can be provided by available free sources - more complex disaster models require much more information, such as soil type and humidity, afforestation, etc. that cannot be provided by free sources. 
Secondly, the application of the model can provide sufficient information to make an economic assessment of the effects of the flood.

The use of more complex models for both floods and other types of disasters is possible if the necessary input geo-referenced information on their application is available. The two major cartographic applications - Mapnik and Overpass API - allow the addition of supplementary information from third sources. The same applies to the platform used for visualization and control $-\mathrm{R}$.

The mathematical model of the flood falls into the so-called model type 1D hydrodynamic model(For full description of the model and its software application see Marzocchi, R., et all (2013) [16]) and uses the following mathematical equations to describe the physical model of the event.

The software model is embedded in the r.inund.fluv[15] application of GRASS GIS.

There are two input data for the model. Vector river and coastline data and raster relief data - Digital Elevation Model (DEM) of the research region.

\subsection{Selecting a Region and a Physical Model}

Having a map of the area, the user should be able to choose a region for the event. Therefore, a software application is required to work with the user (in the case of the system - in the user's browser) to transmit to the server data about the region selected by the client.

In the selected visualization and management framework, Shiny, and its associated $\mathrm{R}$ packages, there is a clear separation between the user and server parts of the applications being developed. The $\mathrm{R}$ leaflet[17] package is used as a client software that allows the user to interactively identify the region of interest.

In this case, the Draw function from the leaflet is used, which can draw a rectangle encompassing that part of the map which the user is willing to explore. Fig. 2 shows how the user can select a region.

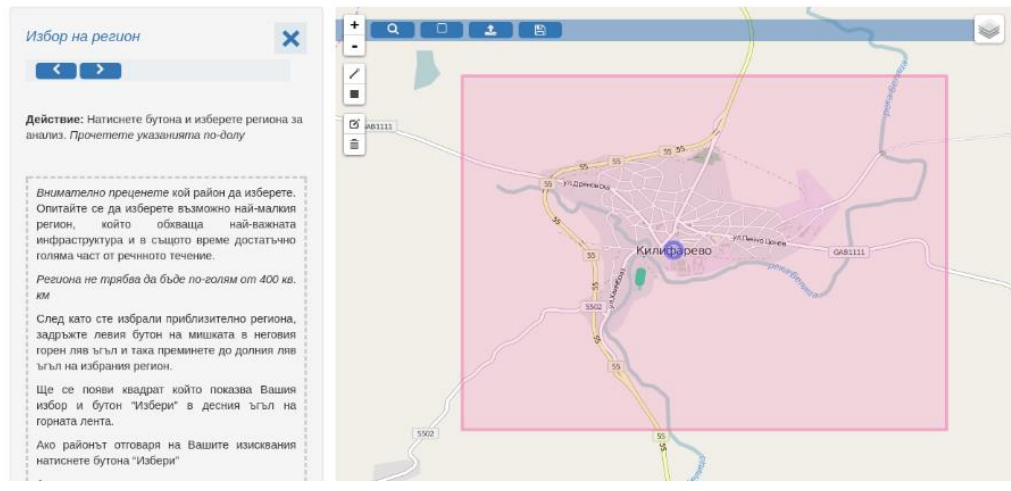

Fig. 2. Selection of a region by the user

Once the region is selected, its coordinates are transmitted to the server of the system. Coordinates are used to submit a request to Overpass API to establish the rivers in 
the region. The result from the request is a vector file containing the river lines that is visualized to the user in the way shown in Fig. 3.

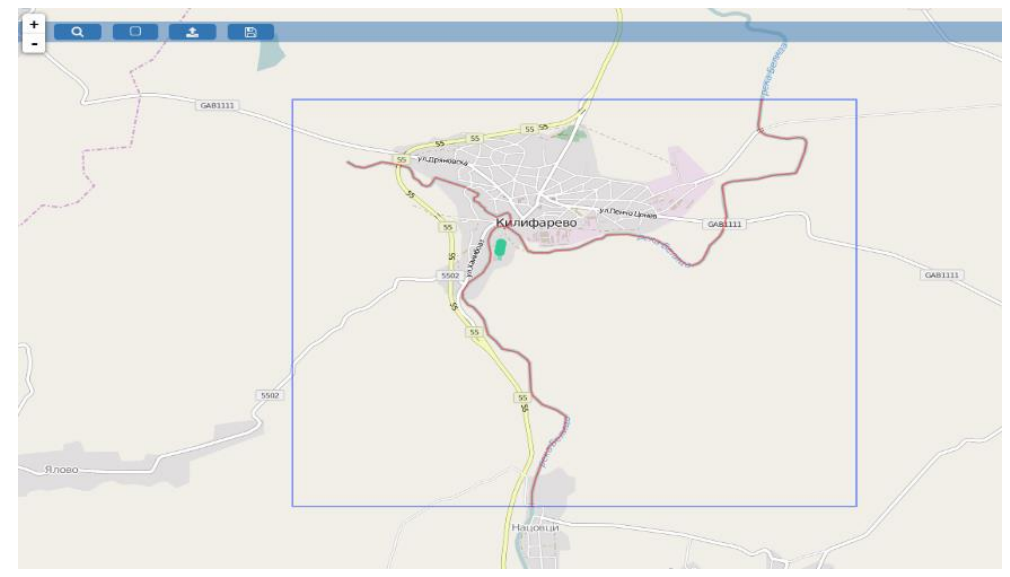

Fig. 3. Visualization of rivers in the region

Following user approval, the following actions are performed in the server part of the system:

- The region is "cut off" the DEM. As a result, a raster file with resolution $27 \times 27$ meters is produced with the size of the region. Due to model requirements, the file is interpolated to a resolution of $3 \times 3$ meters;

- Using the vector file for river lines, reference is made as to the height of the river bed in the raster file of the region. Riverside lines of only the largest rivers are mapped in available vector files. In order to make work easier for the user, the latter will not have to "paint" the shore lines, but an automated coastal calculation is used on the region's raster file using the build_chans() function of the $\mathrm{R}$ dynatopmodel[18].

- Once the required input data is available, the model is implemented by r.inund.fluv from GRASS GIS. The communication between R and GRASS GIS is performed through the spgrass6[19] package from $R$.

The result of the model application is a raster file with the expected depth of the flood. The file is visualized to the user as shown in Fig. 4.

\subsection{Identifying the Affected Infrastructure Sites}

Once we have the flood raster file, it is possible to determine the scope of the flood and thus to identify the affected sites. All objects in the region are established by sending a request to the Overpass API with the coordinates of the region. As a result of the request, we receive a file containing both the coordinates of the objects and information about their type - road, building, type of building, etc. After vectoring the flood raster 
file, we can determine the overlay between the request file and the flood range. This overlay shows us the affected objects.

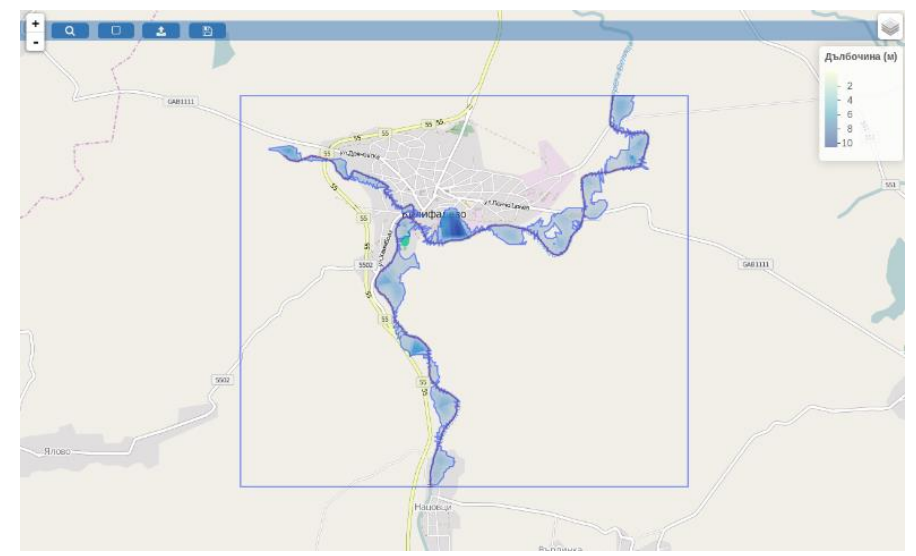

Fig. 4. Results of the model application

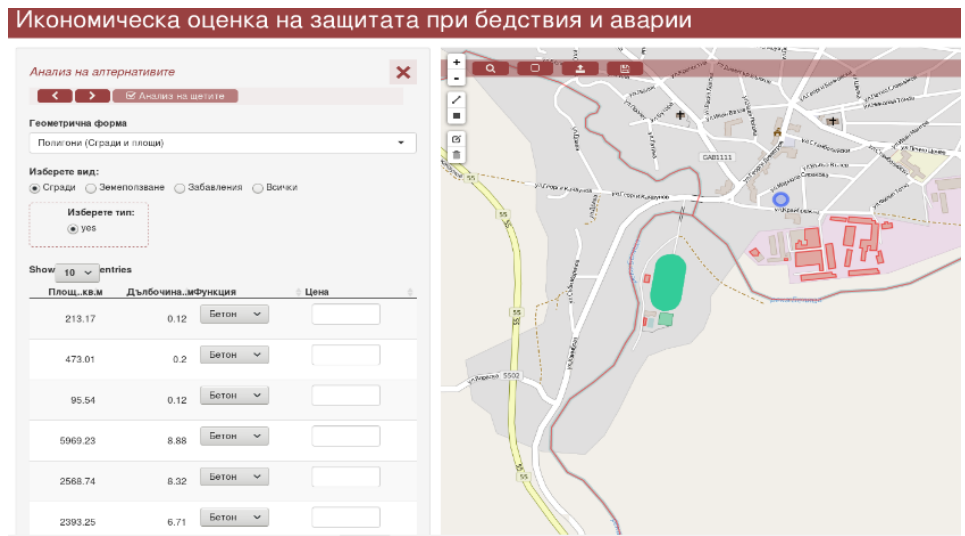

Fig. 5. Objects of the infrastructure in the flood range

The results are visualized to the user in the way shown in Fig. 5. The user already has a list of objects that he can identify according to type and cost.

\subsection{Determination of Direct Economic Losses}

As stated above, the experimental integrated system focuses only on the determination of direct losses from potential disasters and accidents.

The application of the physical flood pattern so far has allowed us to locate the flood area and identify the affected sites. The next step is to determine direct losses as the cost of recovering the affected sites. 
The first approach that can be used here is to determine costs by identifying the intensity of impact of the event on an object and based on this to determine the recovery costs.

This approach has been used by the US Federal Emergency Management Agency (FEMA) to apply models for economic analysis of the consequences of natural disasters in the software application HAZUS[20]. Based on detailed studies of the relationship between flood intensity, on the one hand, and different materials, on the other hand, tables are created to determine the consequences for the relevant sites. Based on a study of average recovery rates for different types of objects, FEMA also sets the values for expected recovery costs at given breakpoints. The intensity of the flood is determined by the depth of the flood, the type of material and the type of the building site. The damage functions dependencies are shown in Fig 6.

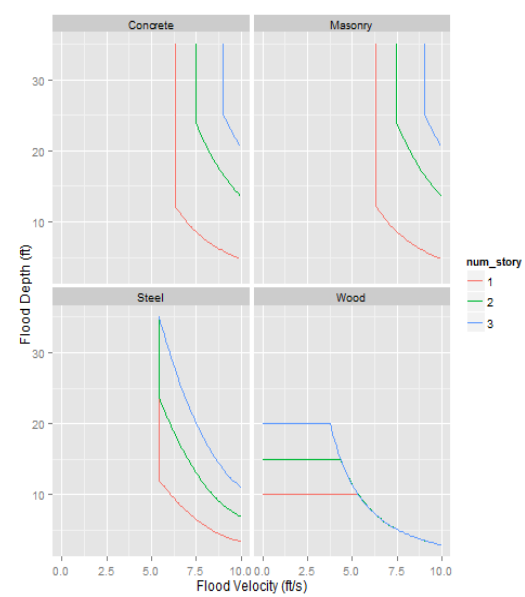

Fig. 6. Dependencies between flood depth, flood velocity and facilities materials

Having the raster file with water depth for each cell (file resolution has cell size 3x3 meters) and taking advantage of the FEMA damage functions for each site, it is possible to determine the cost of flood damage recovery. In this case, they are available for use in $\mathrm{R}$ through the hazus[21] package.

The second possible approach is the direct input of the expected cost of damage recovery based on expert estimates or on the basis of an analysis of the damage from previous events.

The third possible cost estimation option in the integrated system is the use of landuse data from the European Commission's CORINE database[22]. In this geo-referenced database, 44 landuse categories were identified in a raster file with a resolution of approximately 100x100 meters. CORINE landuse reference for the flood area is presented in Fig. 7.

If we have an average estimate of the recovery costs for each category, we can determine the average expected costs for a category based on the flood intensity estimate 
for each cell of the raster file. Such an average estimate is possible in the integrated system and is presented in Fig. 8.

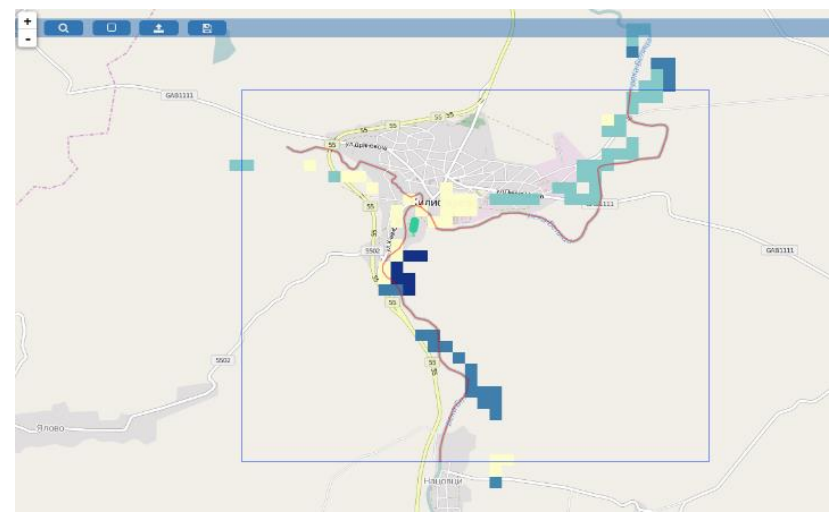

Fig. 7. Categories of affected areas according to CORINE geographic database

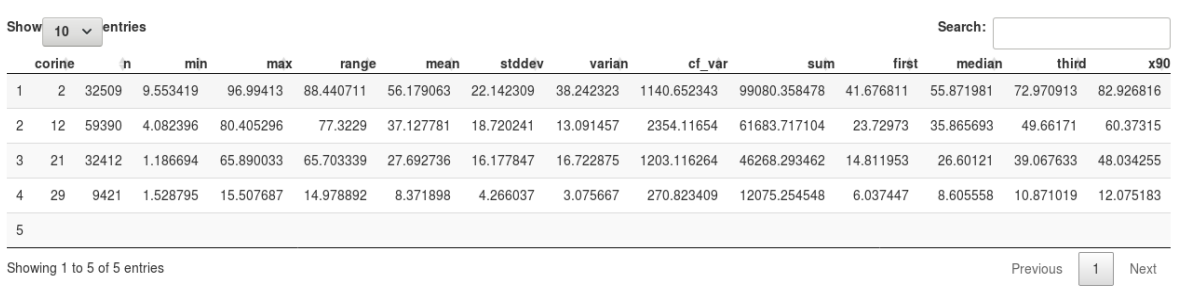

Fig. 8. Table of results on flood intensity broken down by categories of affected areas

The first column of the table presents the identification numbers of the CORINE categories (2 - Discontinuous urban fabric, 12 - Non-irrigated arable land, 21 - Land predominantly occupied by agriculture, 22 - Agro-forestry areas), the remaining columns of the table present the intensity values, such as the number of affected cells, the average depth of the flood, the mean and standard deviation of the flood depth for each category.

Evaluating the three approaches to assess costs deployed in the integrated system, we can see that the third approach requires the least time for assessment and is the most inaccurate, the two more precise approaches require more time to be implemented, as recovery costs need to be determined for the specific region (let us recall that the costs used in the FEMA tables refer to the US regions).

\subsection{Alternatives for Prevention and Protection - Dikes and Canals}

In the experimental integrated system, another module for analysis was introduced - the possibility of generating different alternatives for prevention and protection. In the case of floods, this means changing the terrain in two possible directions - digging rivers or building dikes. 
The change of the terrain is implemented in the basic version by selecting the river line to be excavated and by marking the places for construction of dikes. Fig. 9 presents these options.

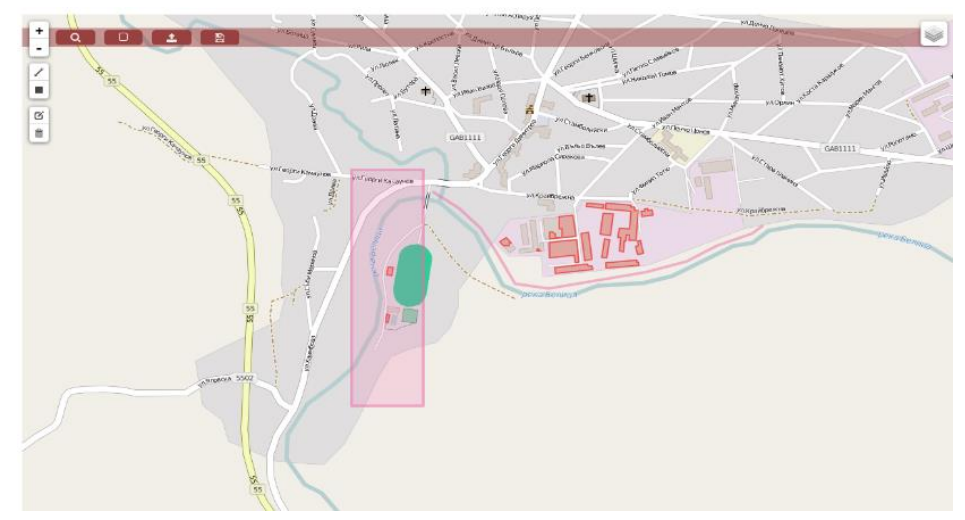

Fig. 9. Setting prevention and protection measures

In the figure, a rectangle is used to help select the part of the river in which a canal is to be dug, and by "drawing" lines the location of dikes to be built is determined. The user has the opportunity to specify a certain width, as well as the depth / height of the canals and dikes.

The lines of the resulting vector file of channels and / or dikes are buffered to the required width by the rgeos[23] application of $\mathrm{R}$. The resulting polygons are used to establish a new overlay with the raster file. Altitude values for cells in the cross section are increased or decreased by the desired value depending on whether they are channels or dikes.

Once the region's DEM has been changed to the user's wishes, the entire workflow of the system is performed again - from the application of the flood model to the damage detection. In this way, the user can quickly identify and compare different prevention and protection alternatives.

\section{Conclusion}

The presented experimental integrated system for assessing economic losses from disasters and accidents largely fulfills the goal of facilitating user work by integrating activities on modeling events, determining economic losses and identifying alternatives to prevention and protection.

At the same time, the following features should be noted in the development of such systems:

Firstly, the task of encompassing a large region within one country and implementing the chosen model in any part of the region greatly complicates the system's operation and requires considerable effort to install and share complex server applications using large volume and complexity of data. 
Secondly, freely available data on flood modeling is sufficient only for the simplest models, with data for economic damage being most difficult to provide.

Last but not least, the development of a working integrated and automated system for economic assessment of disasters and accidents requires previously agreed national norms on the approaches, models and activities for disaster and accident assessment. The availability of such guidance would lead to comparability and stability of the performance of the system.

In the end, it can be stated that even if the results of the work of the proposed system are not accurate with a detailed economic study of costs based on geodesic and geophysical exploration of potential disasters in a given region, the system provides the opportunity for rapid comparison and evaluation across multiple regions, and focusing efforts (further detailed research) on potentially the most dangerous of them.

The ability to reduce expert complexity by automating most of the analytical activities can bring the use of such systems closer to the lower regional and municipal levels of administration, thus providing a cheaper and easier tool to support the rather difficult budget decisions related to the prevention and protection of the population in case of disasters and accidents.

\section{References}

1. OGC® Standards and Supporting Documents, http://www.opengeospatial.org/standards.

2. GRASS GIS - General overview, https://grass.osgeo.org/documentation/general-overview/, last accessed 2017/11/18.

3. GDAL: GDAL - Geospatial Data Abstraction Library, http://www.gdal.org/, last accessed 2017/11/18.

4. Artem Pavlenko, artem at mapnik: Mapnik C++/Python GIS Toolkit, http://mapnik.org/, last accessed 2017/12/01.

5. mod_tile - OpenStreetMap Wiki, https://wiki.openstreetmap.org/wiki/Mod_tile, last accessed 2017/11/29.

6. R Core Team: R: A Language and Environment for Statistical Computing. R Foundation for Statistical Computing, Vienna, Austria (2017).

7. Chang, W., Cheng, J., Allaire, J.J., Xie, Y., McPherson, J.: shiny: Web Application Framework for R. (2017).

8. Shiny Server, https://www.rstudio.com/products/shiny/shiny-server/, last accessed $2017 / 11 / 18$

9. Nominatim - OpenStreetMap Wiki, https://wiki.openstreetmap.org/wiki/Nominatim, last accessed 2017/11/18.

10. Technical Overview, http://overpass-api.de/technical_overview.html, last accessed 2017/11/29.

11. Geographical information system of the Commission (GISCO) - Statistics Explained, http://ec.europa.eu/eurostat/statistics-explained/index.php/Geographical_information_system_of_the_Commission_(GISCO), last accessed 2017/12/02.

12. USGS.gov, Science for a changing world, https://www.usgs.gov/, last accessed 2017/12/02.

13. ESDAC - European Commission, https://esdac.jrc.ec.europa.eu/, last accessed 2017/12/01.

14. COPERNICUS, Emergency Management Service, http://emergency.copernicus.eu/, last accessed 2017/12/02. 
15. Ferrando, I., Federici, B., Sguerso, D., Marzocchi, R.: The r. inund. fluv tool for flood-prone areas evaluation in GRASS GIS: application to the terminal reach of Magra River. Geomat. Workb. 12 FOSS4G Eur. Como. (2015).

16. Marzocchi, R., Federici, B., Cannata, M., Cosso, T., Syriou, A.: The Contribution of GIS in Flood Mapping: Two Approaches Using Open Source Grass GIS Software. ISPRS - Int. Arch. Photogramm. Remote Sens. Spat. Inf. Sci. 175-178 (2013).

17. Cheng, J., Karambelkar, B., Xie, Y.: leaflet: Create Interactive Web Maps with the JavaScript "Leaflet" Library. (2017).

18. Metcalfe, P., Beven, K., Freer, J.: dynatopmodel: Implementation of the Dynamic TOPMODEL Hydrological Model. (2018).

19. Bivand, R.: spgrass6: Interface between GRASS 6 and R. (2016).

20. Hazus, FEMA.gov, https://www.fema.gov/hazus, last accessed 2017/12/03.

21. Goteti, G.: hazus: Damage functions from FEMA's HAZUS software for use in modeling financial losses from natural disasters. (2014).

22. CORINE Land Cover, https://www.eea.europa.eu/publications/COR0-landcover, last accessed 2017/12/03.

23. Bivand, R., Rundel, C.: rgeos: Interface to Geometry Engine - Open Source ('GEOS'). (2017). 